\title{
Verbal fluency in Huntington's disease: a longitudinal analysis of phonemic and semantic clustering and switching
}

\author{
Aileen K. Ho ${ }^{\text {a } *}$, Barbara J. Sahakian ${ }^{b}$, Trevor W. Robbins ${ }^{c}$, Roger A. Barker ${ }^{\text {a }}$, \\ Anne E. Rosser ${ }^{\mathrm{a}}$, John R. Hodges ${ }^{\mathrm{d}}$ \\ ${ }^{a}$ Cambridge Centre for Brain Repair, University of Cambridge, Forvie Site, Robinson Way, Cambridge CB2 2PY, UK \\ ${ }^{\mathrm{b}}$ Department of Psychiatry, University of Cambridge, Addenbrooke's Hospital, Cambridge CB2 2QQ, UK \\ ${ }^{\mathrm{c}}$ Department of Experimental Psychology, University of Cambridge, Cambridge CB2 2PP, UK \\ ${ }^{\mathrm{d}}$ MRC Cognition and Brain Sciences Unit, Cambridge CB2 2EF, UK
}

Received 24 August 2001; received in revised form 12 November 2001; accepted 12 November 2001

\begin{abstract}
Two underlying components of verbal fluency tasks have been identified as clustering (the ability to generate successive words within a sub-category) and switching (the ability to shift from one sub-category to another). Selective impairment of switching ability occurs in patients with frontostriatal pathology, whilst clustering ability is compromised with temporal lobe dysfunction. Letter fluency tasks have been shown to be especially sensitive to frontostriatal deficits, whereas, category fluency tasks tend to be compromised by temporal lobe pathology. This study examined two types of verbal fluency task (letter fluency and category fluency) using two levels of analysis (phonemic and semantic) for clustering and switching measures. The performance of 21 frontostriatally compromised Huntington's disease (HD) patients was followed over an average of 3.5 annual follow-up visits. HD patients showed a significant reduction of correctly generated words over time, together with a significant increase in word repetitions. Phonemic switching decreased significantly over time for both letter and category fluency. Semantic switching, however, remained stable over time for both verbal fluency tasks. Clustering (both semantic and phonemic) likewise remained stable and did not vary longitudinally for either letter or category fluency. Hence, phonemic switching alone drove verbal fluency performance and this selective impairment can be explained by the progressive involvement of frontostriatal circuitry in the natural progression of HD. (c) 2002 Elsevier Science Ltd. All rights reserved.
\end{abstract}

Keywords: Frontostriatal; Neuropsychology; Cognition; Response set; Motor; Functional assessment

\section{Introduction}

Verbal fluency is a commonly used neuropsychological test which examines the ability to spontaneously produce words orally within a fixed time span. There are two types of verbal fluency tasks, i.e. letter fluency (variously known in the literature as initial letter fluency, phonemic fluency, phonological fluency, formal fluency, letter-cue word generation) and category fluency (or semantic (category) fluency, semantic-cue word generation). For letter fluency, words must be produced according to phonemic constraints (i.e. exemplars beginning with a specified letter of the alphabet such as ' $\mathrm{f}$ '). For category fluency, words must be produced according to semantic constraints (i.e. exemplars which belong to a specified semantic category, such as 'animal'). The measure of performance most commonly used is the number of correctly generated words within $60 \mathrm{~s}$. This measure

* Corresponding author. Tel.: +44-1223-331160; fax: +44-1223-331174.

E-mail address: aileenkho@netscape.net (A.K. Ho). is believed to tap frontal $[9,10]$ and temporal $[8,11,24]$ lobe function and is generally decreased in Huntington's disease (HD) patients relative to controls [5-7,36]. Indeed, there is some evidence for a decline in performance over time for letter [17] and category fluency in HD [2], despite the lack of cross-sectional difference for both letter and category fluency when comparing early versus late HD $[5,6]$.

A qualitative examination of the sequence and nature of the actual words generated provides further insights on the different types of processes that occur in this task. Observation of clustering (where related words are produced rapidly one after the other) and switching (where there is a slight pause before another cluster of related words is produced) has led to the proposition that two distinct but complementary cognitive strategies (i.e. clustering and switching) are in operation during verbal fluency tasks $[14,37,40]$. This concept has been refined and operationalised by Troyer et al. [37] and subsequently used to investigate further the nature of verbal fluency performance in neurologically impaired patients $[31,36]$. Thus, the extent to which patients demonstrate 
clustering which involves either a phonemic (e.g. words beginning with 'fa_' or 'fi_') or semantic (e.g. farm animals or safari animals) sub-category search, and switching which involves a shift to a new sub-category (phonemic or semantic) can be compared to yield further insights into the possible pathophysiological substrates critical for performance of verbal fluency tasks.

The letter fluency task has been shown to be especially sensitive to frontostriatal pathology, such as that following PSP [33] and frontal lobe lesions [9,29]. Category fluency tasks tend to be compromised by temporal lobe deficits seen in Alzheimer's disease [19,27,33] and particularly in semantic dementia $[18,20]$. This difference may be explained by the type of search necessary to perform the fluency tasks. The category fluency task utilises a more familiar search strategy because of its reliance on meaning, where activation of the first prototypical exemplar automatically activates other semantically related words [22]. The letter fluency task, on the other hand, is based on the more artificial level of word representation rather than meaning and prompts a less familiar phonological search strategy through the mental lexicon to find novel category neighbours [33]. Another interesting finding emerging from the literature is that switching tends to be reduced in frontal patients [38], frontostriatal disorders (e.g. PD with dementia patients [39]) and even in a divided attention paradigm which simulates frontal dysfunction in healthy participants [37]. Clustering, however, is impaired in patients with temporal pathology [38] and with Alzheimer's disease [39]. Whilst this frontostriatal-temporal dichotomy is not always clear cut [36], it appears that brain pathology has a systematic impact on the type of cognitive process that becomes compromised in verbal fluency tasks.

Verbal memory tasks require the use of working memory to operationalise the cue and track the exemplars generated. Several other processes are common among fluency tasks, such as the direction of attention to the task, implementation of appropriate search strategies, word retrieval and finally articulation of the word [26]. However, different search strategies are employed. For the letter fluency task, a primarily phonemic search is utilised; a phonemic switching strategy is instrumental for high performance because of the many sub-categories available, e.g. by phonemically varying the stem component to generate words that begin with a particular initial letter (such as $\mathrm{sa}_{-}, \mathrm{se}_{-}$, si_, $\mathrm{so}_{-}, \mathrm{su}_{-}, \mathrm{sc}_{-}, \mathrm{sh}_{-}, \mathrm{etc}$.). Due to the vast number of alphabetic permutations as well as the short $60 \mathrm{~s}$ duration of the task, the clustering strategy is comparatively less important [36]. For the category fluency task, however, a primarily semantic search takes place and is fundamentally hierarchical as it emphasises a semantic clustering strategy which looks for many same-level exemplars of a rather broad semantic sub-categories (e.g. farm animals, safari animals, jungle animals, reptiles, etc.). However, the semantic switching strategy is also utilised when a semantic sub-category is exhausted and, therefore, both switching and clustering are important [36].
In the earlier stages of $\mathrm{HD}$, pathology begins at the dorsal caudate nucleus which is part of the dorsolateral prefrontal cortex loop and gradually spreads throughout the frontostriatal system, with only minimal impairment of the temporal lobes [16]. From this, several hypotheses emerge regarding the change in performance of verbal fluency tasks over the natural progression of the disease. Firstly, the use of the more frontally-mediated phonemic switching strategy important in letter fluency performance may be progressively reduced in HD patients due to increasing frontostriatal involment over time. Secondly, use of the more temporal lobe dependent semantic clustering strategy instrumental in the category fluency task may remain stable. Thirdly, it will be of interest to examine if there is also a progressive phonemic switching deficit in category fluency, where a primarily semantic search strategy is implemented. In other words, whether a generalised phonemic switching impairment occurs as the disease progresses.

Only the first hypothesis has been partially investigated in a study of longitudinal performance of HD patients in a letter fluency task. Rich et al. [31] found a deterioration of phonemic switching on the letter fluency task over time in HD patients but no reduction in clustering on the same task. However, to test the generality of the findings, category fluency must be examined as well. Therefore, this study aims to address this for the first time by examining the pattern of deterioration in clustering and switching ability by conducting phonemic and semantic analyses for both letter and category fluency tasks, over the course of longitudinal testing in patients with HD.

\section{Methods}

\subsection{Participants}

Twenty one patients (15 males and six females) with mild to moderate $\mathrm{HD}$ at baseline were followed longitudinally via yearly assessments. All these patients had a positive family history of $\mathrm{HD}$ and more than $36 \mathrm{CAG}$ repeats upon genetic testing for the IT-15 mutation [15]. They all gave written consent to participate in this ethically approved study. At baseline, the patients' mean age was 47.8 years $($ S.D. $=10.6)$ and they scored an average of 28.4 (S.D. $=1.7$ ) out of 30 on the mini mental state examination (MMSE), 110.3 (S.D. $=9.5$ ) on the national adult reading test (NART) and 24.7 (S.D. $=14.2)$ for the unified HD rating scale (UHDRS) total motor score. As a result of the progressive nature of ongoing enrolment to the cohort, the number of follow-up assessments post-baseline varied but overall patients were followed up for an average of 3.5 (S.D. $=1.1)$ annual assessments.

\subsection{Procedure}

At annual assessments, patients performed two verbal fluency tasks. Firstly, the letter fluency task where they were 
required to orally generate as many words (excluding proper nouns and variants of the same root word) which began with the letters ' $f$ ', ' $a$ ' and 's' for $60 \mathrm{~s}$ each [4]. Secondly, for the category fluency task, patients were required to name as many animals as they could within a $60 \mathrm{~s}$ duration [7]. All responses were recorded verbatim. The MMSE [13] and UHDRS motor examination [21] were also administered at each yearly assessment. For the most recent (endpoint) assessment, several other neuropsychological tests sensitive to frontostriatal impairment in HD were undertaken including the intra/extra dimensional (IDED) set-shifting task of the Cambridge neuropsychological test automated battery (CANTAB) [12], Stroop test [34] and Reitan trail-making test [30] to examine the relationship between these tests and the verbal fluency measures investigated in this study.

\subsection{Scoring method}

For both the letter and category fluency tasks the typical quantitative measure of total number of correct words within $60 \mathrm{~s}$ was scored. Additionally, four qualitative measures were made for each verbal fluency task. These measures comprised two types (i.e. phonemic and semantic) of clustering and switching scores as described below:

\section{Phonemic clustering score}

Clusters were defined as sequences of words which began with either (a) at least the same first two letters (verbal fluency, e.g. fan, fast, family; category fluency, e.g. cat, camel, caterpillar), (b) words which differed by rhyme or only by one phoneme (verbal fluency, e.g. fan, fin, fun; category fluency, e.g. cat, rat, bat) or (c) were homophones (verbal fluency, e.g. sole, soul; does not occur in category fluency).

2. Semantic clustering score

Clusters were defined as sequences of words which belong to the same semantic sub-category (verbal fluency, e.g. swim, sand, snorkel-all to do with the seaside; category fluency, e.g. cow, pig, goat-all farm animals).

3. Phonemic switching score

Phonemic switches were defined as the number of transitions between phonemic clusters, including single words (verbal fluency, e.g. 'fat, fall, fit' has one phonemic switch, whilst 'far, farther, farthing' has no phonemic shift; category fluency, e.g. 'ant, antelope, fish' has one phonemic shift, while 'zebra, lion, tiger' has two phonemic shifts).

4. Semantic switching score

Semantic switches were defined as the number of transitions between semantic clusters, including single words (verbal fluency, e.g. 'fat, fall, fit' has two semantic switches, whilst 'far, farther, farthing' one semantic shift; category fluency, e.g. 'ant, antelope, fish' has two semantic shifts, while 'zebra, lion, tiger' has no semantic shifts).
These scoring rules were devised by Troyer et al. [37] (refer to their Appendix for further details and scoring examples), where errors and repetitions were included in the analysis of the four qualitative scores above, but not for the total correct score for the letter fluency and category fluency tasks. This is because, while it is not valid to include errors for the quantitative measure of correctly produced words, errors may arise from a deficit in the underlying cognitive processes under scrutiny and should, therefore, be captured by the more qualitative analysis of clustering and switching scores. Larger clustering scores reflect increased cluster size, whilst larger switching scores a higher frequency of switches. These indices have been shown to have high interrater reliabilities (i.e. $r>0.90$ ) [37].

\subsection{Analysis}

To maximise the inclusion of data collected for each patient and to minimise the variability that is common in this neurological group, performance over time was quantified by the means of calculating regression slopes utilising each annual assessment data point available for each individual patient. The assumption of linear decline is relevant and has been previously used for this population [23].There were at least three data points (yearly assessments) for any patient, hence yielding a robust regression line to depict the picture of possible deterioration, improvement or stable performance over time. The effect sizes for the mean of patients' regression scores for each measure was then tested for significance with $\alpha$ at 0.05 (two-tailed). As patients' successive performances over time was of interest, a healthy control group was not used [2,3].

A correlational analysis was performed between switching and clustering measures on both fluency tasks with neurological indices and other neuropsychological tests which are implicated in the concept of mental set switching and are known to be sensitive to frontal dysfunction. Spearman's correlation coefficients were computed based on patients' raw scores at their most recent assessment, respectively. End point rather than baseline scores were used in order to obtain a greater range of performance on each measure. As this was an exploratory analysis, a non-conservative approach was adopted and adjustment for multiple comparisons was not made in order to avoid type 1 error. However, exact levels of significance are reported in the text.

\section{Results}

For both letter and category fluency, HD patients showed a significant reduction of correct words over time (letter fluency mean regression slope $=-0.10, P=0.039$; category fluency mean regression slope $=-0.08, P<0.005)$, together with a significant increase in repetitions (letter fluency mean regression slope $=0.03, P<0.050$; category fluency mean regression slope $=0.01, P<0.010$ ). 
a) Letter fluency task: phonemic clustering

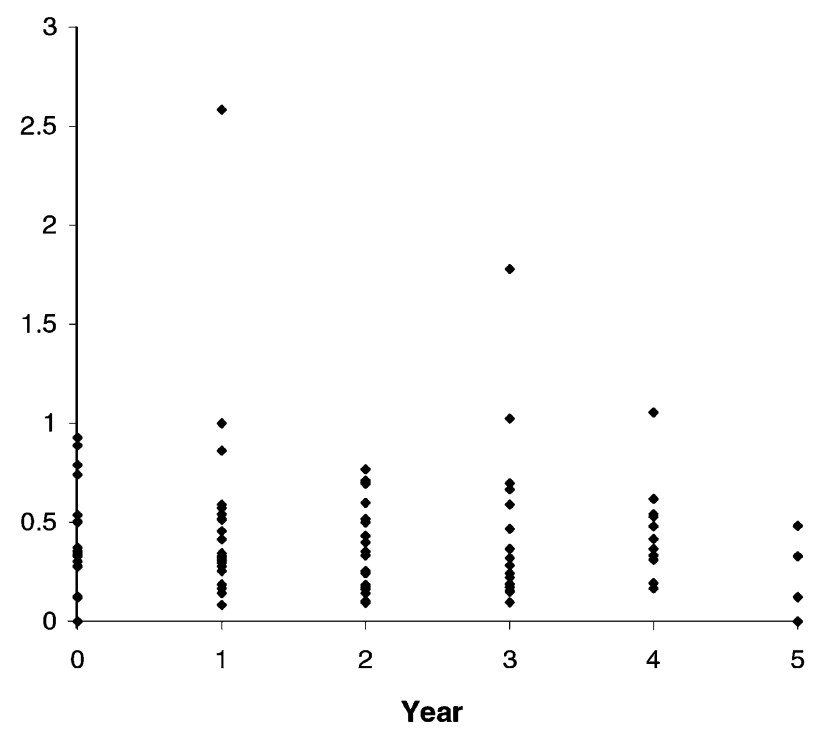

b) Letter fluency task: phonemic switching

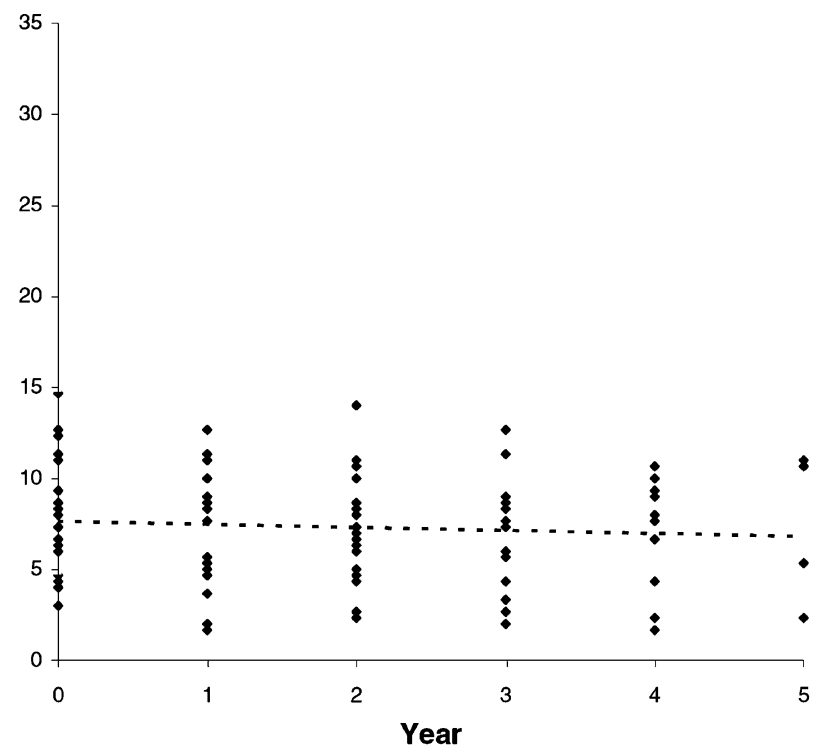

c) Letter fluency task: semantic clustering

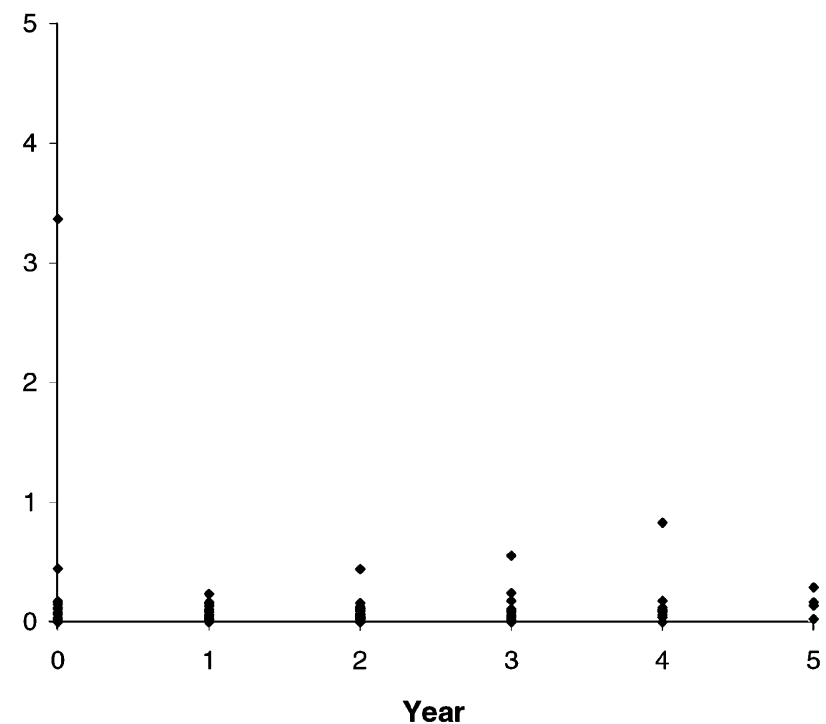

d) Letter fluency task: semantic switching

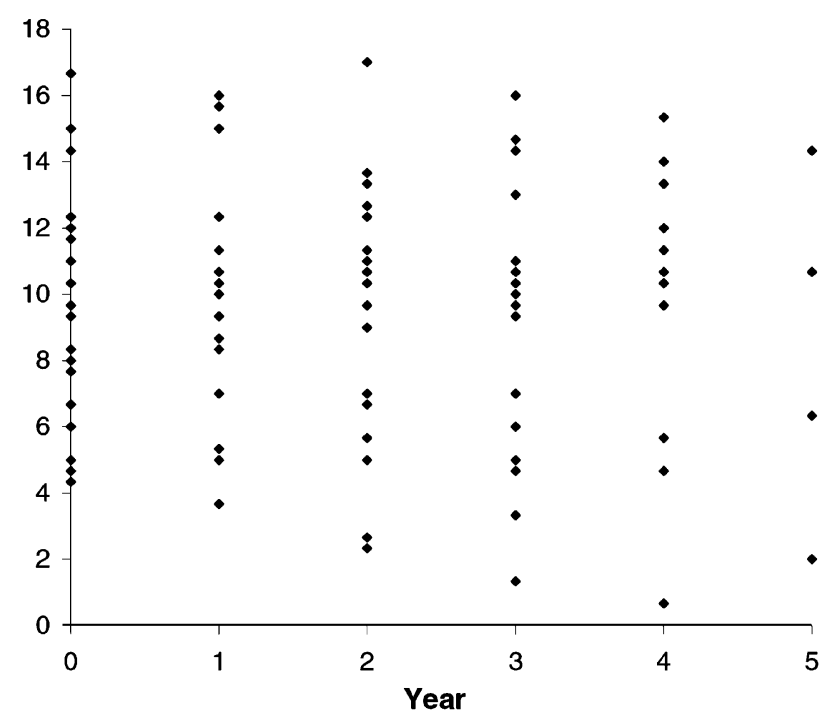

Fig. 1. Scatter plot of scores for (phonemic and semantic) clustering and switching for the letter fluency task.

Letter fluency errors, however, decreased over time (mean regression slope $=-0.03, P=0.044$ ).

Figs. 1 and 2 illustrate individual HD patients' phonemic and semantic clustering and switching for the letter fluency and category fluency tasks. Generally, it shows that semantic clustering occurred more frequently for the semantic task and phonemic clustering for the phonemic letter fluency task. The regression slope for each patient's performance on each of the measures was obtained and from this the means were obtained and presented in Table 1. For measures where there was a significant change over time (phonemic switching in the letter fluency task and phonemic switching in the category fluency task), the mean regression slope for that measure was superimposed on the relevant scatter plot of scores (Figs. 1b and 2b, respectively). A key finding was that phonemic switching decreased significantly over time for letter (mean regression slope $=-0.06, P=0.050$ ) and for category fluency (mean regression slope $=-0.08, P=$ 0.001). Semantic switching however, remained stable over time for both fluency tasks. Likewise, clustering (semantic and phonemic) did not vary longitudinally for both letter and category fluency.

Spearman's correlation coefficients performed on patients' last assessment scores (end point data) are presented in Table 2. From this analysis, motor performance (UHDRS total: $r=-0.511, P=0.018$; chorea: $r=$ 
a) Category fluency task: phonemic clustering

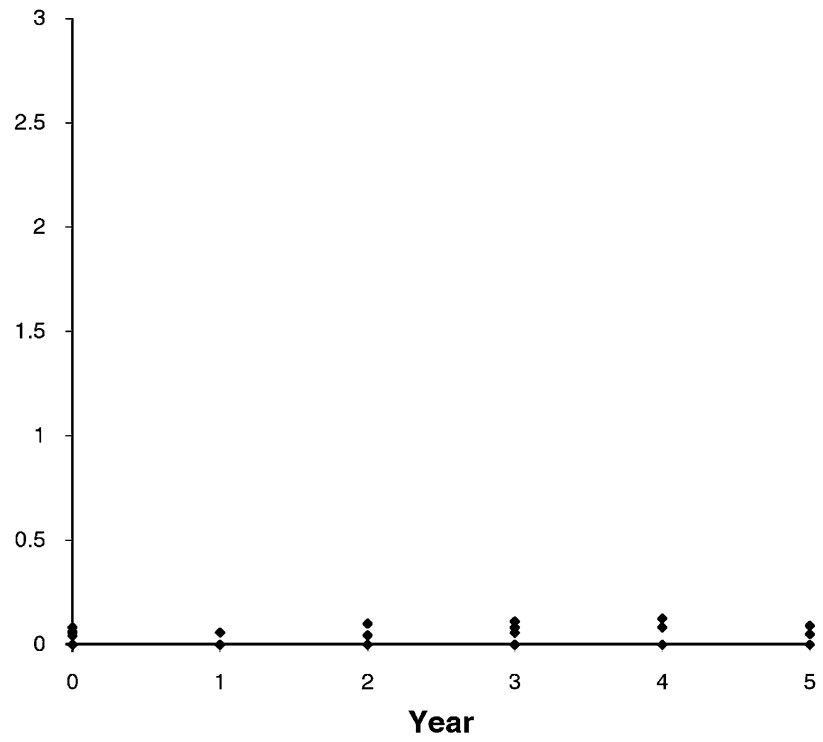

b) Category fluency task: phonemic switching

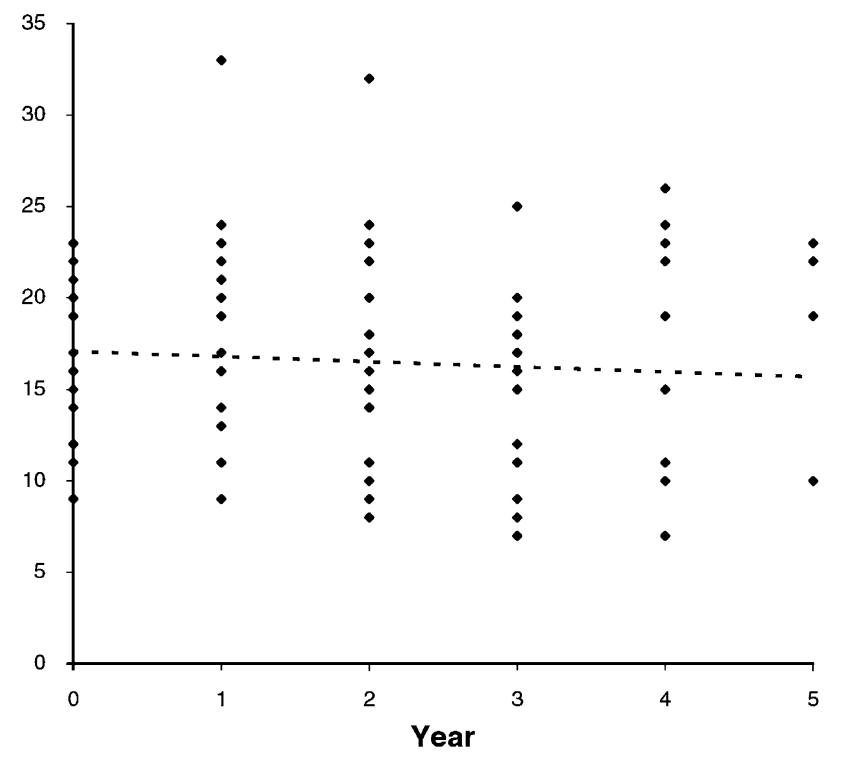

c) Category fluency task: semantic clustering

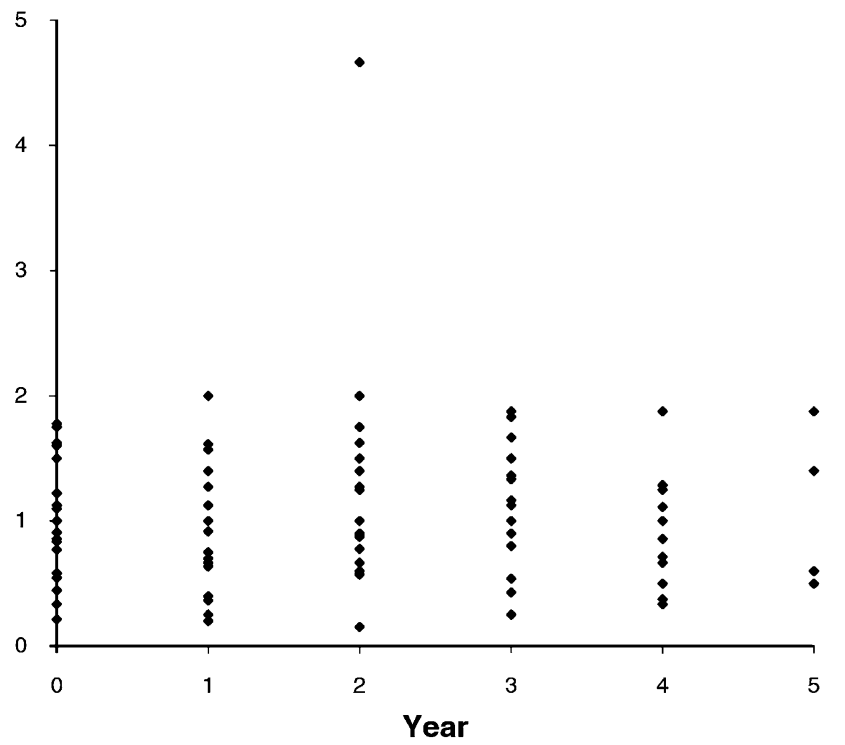

d) Category fluency task: semantic switching

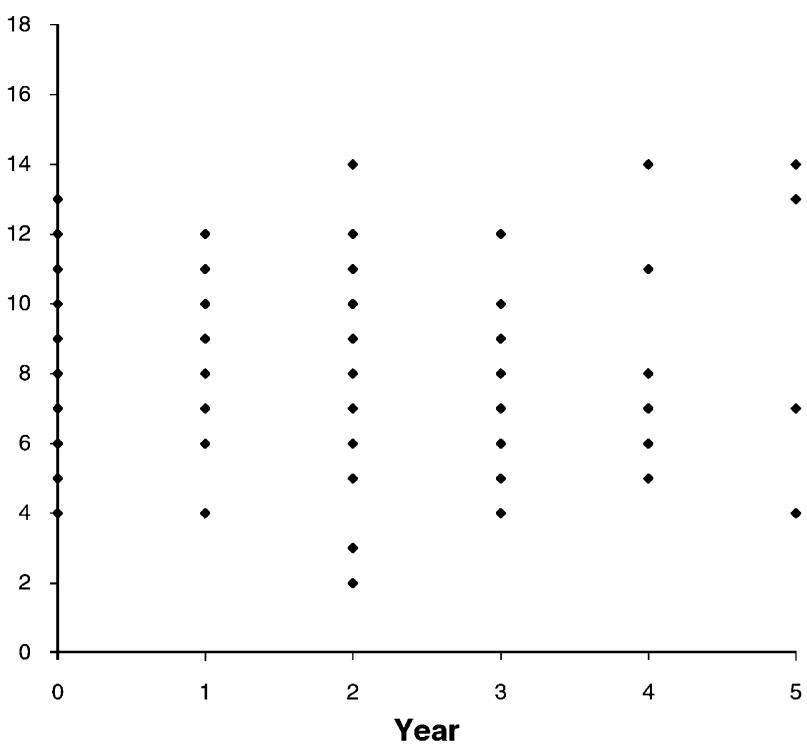

Fig. 2. Scatter plot of scores for (phonemic and semantic) clustering and switching for the category fluency task.

$-0.558, P=0.009$; bradykinesia: $r=-0.467, P=0.033$; total functional score: $r=-0.443, P=0.045)$ correlated with phonemic switching on the letter fluency task, such that low motor performance was related to low phonemic switching. Functional impairment in everyday living (total functional score: $r=-0.442, P=0.046$; functional capacity: $r=-0.451, P=0.040)$ correlated with phonemic clustering in the letter fluency task such that impaired performance in one measure was associated with impaired performance in the other measure. General cognition as measured by the MMSE ( $r=0.447, P=0.029$ ) correlated positively with the ability to perform semantic switching in the animal fluency task such that high MMSE scores were associated with high switching ability.

Both primary switching measures for the letter (phonemic switching) and animal (semantic switching) fluency tasks correlated with neuropsychological tasks which are influenced by frontal lobe functioning, such as the IDED set switching task, Stroop test and Reitan trail-making test. Specifically, phonemic switching for the letter fluency task correlated with the IDED task total error score $(r=-0.468$, $P=0.032)$, Stroop colour naming $(r=0.553, P=0.009)$, Stroop word naming $(r=0.591, P=0.005)$, Stroop interference $(r=0.582, P=0.006)$, trails A $(r=-0.485$, 
Table 1

Mean regression slopes on verbal fluency measures

\begin{tabular}{|c|c|c|c|c|}
\hline & Mean regression slope & Standard error & Effect size & $P$ value (two-tailed) \\
\hline UHDRS total motor score & 0.39 & 0.04 & 9.20 & $<0.005^{*}$ \\
\hline MMSE & -0.01 & 0.01 & -0.55 & 0.582 \\
\hline \multicolumn{5}{|l|}{ Letter fluency } \\
\hline Total score & -0.10 & 0.05 & -2.08 & $0.039 *$ \\
\hline Total errors & -0.03 & 0.01 & -2.02 & $0.044^{*}$ \\
\hline Total repetitions & 0.03 & 0.01 & 2.17 & $0.030^{*}$ \\
\hline Phonemic clustering & 0.00 & 0.00 & 1.54 & 0.124 \\
\hline Phonemic switching & -0.06 & 0.03 & -1.96 & $0.050^{*}$ \\
\hline Semantic clustering & -0.01 & 0.01 & -1.18 & 0.242 \\
\hline Semantic switching & -0.04 & 0.03 & -1.43 & 0.153 \\
\hline \multicolumn{5}{|l|}{ Category fluency } \\
\hline Total score & -0.08 & 0.02 & -3.54 & $<0.005^{*}$ \\
\hline Total errors & 0.00 & 0.00 & -0.98 & 0.327 \\
\hline Total repetitions & 0.02 & 0.01 & 2.46 & $0.014^{*}$ \\
\hline Phonemic clustering & 0.00 & 0.00 & 0.01 & 0.992 \\
\hline Phonemic switching & -0.08 & 0.03 & -2.58 & $0.010^{*}$ \\
\hline Semantic clustering & 0.00 & 0.00 & -0.40 & 0.689 \\
\hline Semantic switching & -0.03 & 0.02 & -1.36 & 0.174 \\
\hline
\end{tabular}

* Significant at $P<0.05$.

$P=0.026)$ and trails B $(r=0.471, P=0.031)$. Semantic switching for the animal fluency task correlated with the IDED task extra dimensional shift error $(r=0.444, P=$ 0.044), Stroop colour naming $(r=0.584, P=0.005)$, Stroop word naming $(r=0.582, P=0.006)$, Stroop interference $(r=0.474, P=0.030)$ and trails B $(r=0.495$, $P=0.023)$.
Secondary switching measures for the letter (semantic switching) and animal (phonemic tasks) fluency tasks also correlated with frontal neuropsychological tasks but on fewer measures. Specifically, semantic switching on the letter fluency task correlated with Stroop colour naming $(r=0.503, P=0.020)$, Stroop word naming $(r=$ $0.601, P=0.004)$ and Stroop interference $(r=0.509$,

Table 2

Correlations between (phonemic and semantic) clustering and switching measures with key neurological and neuropsychological indices

\begin{tabular}{|c|c|c|c|c|c|c|c|c|}
\hline & \multicolumn{4}{|c|}{ Letter fluency task } & \multicolumn{4}{|c|}{ Category fluency task } \\
\hline & $\begin{array}{l}\text { Phonemic } \\
\text { clustering }\end{array}$ & $\begin{array}{l}\text { Phonemic } \\
\text { switching }\end{array}$ & $\begin{array}{l}\text { Semantic } \\
\text { clustering }\end{array}$ & $\begin{array}{l}\text { Semantic } \\
\text { switching }\end{array}$ & $\begin{array}{l}\text { Phonemic } \\
\text { clustering }\end{array}$ & $\begin{array}{l}\text { Phonemic } \\
\text { switching }\end{array}$ & $\begin{array}{l}\text { Semantic } \\
\text { clustering }\end{array}$ & $\begin{array}{l}\text { Semantic } \\
\text { switching }\end{array}$ \\
\hline \multicolumn{9}{|l|}{ Neurological indices } \\
\hline \multicolumn{9}{|l|}{ UHDRS } \\
\hline Total motor score & 0.25 & $-0.51^{*}$ & 0.16 & -0.39 & 0.26 & -0.39 & -0.04 & -0.35 \\
\hline Rigidity & 0.05 & -0.41 & 0.27 & -0.38 & 0.35 & -0.35 & -0.16 & -0.30 \\
\hline Chorea & 0.32 & $-0.56^{*}$ & 0.05 & -0.41 & 0.15 & -0.41 & 0.11 & -0.38 \\
\hline Bradykinesia & 0.19 & $-0.47^{*}$ & 0.21 & -0.38 & 0.27 & -0.37 & -0.06 & -0.34 \\
\hline Total functional score & $0.44^{*}$ & $-0.44^{*}$ & -0.02 & -0.30 & -0.11 & -0.42 & -0.08 & -0.43 \\
\hline Independence scale & -0.29 & 0.30 & -0.04 & 0.18 & 0.01 & 0.30 & 0.13 & 0.31 \\
\hline Functional capacity & $-0.45^{*}$ & 0.33 & 0.04 & 0.19 & 0.04 & 0.26 & 0.16 & 0.24 \\
\hline \multicolumn{9}{|l|}{ Neuropsychological indices } \\
\hline MMSE & -0.02 & 0.34 & -0.25 & 0.39 & -0.05 & 0.38 & -0.11 & $0.48^{*}$ \\
\hline \multicolumn{9}{|l|}{ IDED test } \\
\hline Errors at ED stage & 0.29 & -0.15 & 0.00 & -0.10 & -0.08 & -0.15 & 0.24 & $-0.44^{*}$ \\
\hline Latency at ED stage & 0.32 & -0.07 & 0.20 & -0.06 & 0.01 & -0.04 & -0.30 & 0.07 \\
\hline Average errors (all stages) & 0.35 & $-0.47^{*}$ & 0.11 & -0.39 & -0.08 & -0.23 & 0.05 & -0.35 \\
\hline Average latency (all stages) & 0.32 & -0.11 & -0.05 & -0.07 & 0.01 & -0.18 & -0.37 & -0.04 \\
\hline \multicolumn{9}{|l|}{ Stroop test } \\
\hline Colour naming & -0.19 & $0.55^{*}$ & -0.13 & $0.50^{*}$ & -0.06 & $0.57^{*}$ & -0.03 & $0.58^{*}$ \\
\hline Word reading & 0.11 & $0.59^{*}$ & -0.22 & $0.60^{*}$ & -0.18 & $0.51^{*}$ & -0.04 & $0.58^{*}$ \\
\hline Interference & -0.11 & $0.58^{*}$ & -0.24 & $0.51^{*}$ & -0.021 & $0.46^{*}$ & -0.03 & $0.47^{*}$ \\
\hline \multicolumn{9}{|l|}{ Reitan trail-making test } \\
\hline Trails A & 0.17 & $-0.48^{*}$ & 0.05 & -0.40 & 0.08 & -0.30 & 0.15 & -0.41 \\
\hline Trails B & 0.205 & $-0.47^{*}$ & -0.01 & -0.38 & 0.00 & -0.40 & 0.14 & $-0.49^{*}$ \\
\hline
\end{tabular}

* Significant at $P<0.05$. 
$P=0.019)$. Phonemic switching on the semantic fluency task correlated with Stroop colour naming $(r=0.567, P=$ 0.007), Stroop word naming $(r=0.507, P=0.019)$ and Stroop interference $(r=0.459, P=0.036)$.

\section{Discussion}

This study examined the longitudinal performance of HD patients on measures of verbal fluency over an average duration of 3.5 annual assessments. A quantitative analysis of the total number of correctly produced words showed that the patients score deteriorated over time on both letter and category fluency tasks and they also produced more repetitions. This finding is consistent with the role of the frontal lobe in tests of verbal fluency $[2,9,10,17]$ and provides further evidence that longitudinal deterioration within HD individuals occurs, even though this is not evident from studies utilising cross-sectional paradigms $[5,6]$.

A qualitative scrutiny of the sequence of words generated showed that as predicted, phonemic switching (but not clustering) deteriorated over time in the letter fluency task, consistent with Rich et al. [31]. Furthermore, this progressive impairment in phonemic switching also occurred in the category fluency task, again in the absence of any progressive clustering impairment. The consistency of a progressive switching impairment across letter and category fluency extends Rich's [31] findings and provides further evidence for a deterioration in cognitive flexibility. The ability to freely switch between sub-categories in order to generate appropriate exemplars is crucial in the performance of these time-constrained fluency tasks. Progressive impairment to the switching process, in contrast to stable clustering, is entirely consistent with the disruption of the frontostriatal circuit in HD [31,37]. HD patients' ability to utilise a clustering strategy for phonemic and semantic searches are not compromised and neither is the capacity for switching semantic sub-categories. It appears that HD patients' letter and category fluency performance gradually declined because they are unable to switch to different phonemic sub-categories in order generate more words. Phonemic searches and as a consequence phonemic switching may be considered more artificial, less automatic and, therefore, more frontally-dependent in contrast to semantic searches which involve regions of the brain implicated with representation of semantic knowledge, notably the temporal lobes $[22,26,33]$. Reliance of fluency performance upon frontostriatal (particularly the dorsolateral prefrontal) areas has been demonstrated in recent imaging studies $[1,35]$. The impairment in switching between phonemic sub-categories could be construed as an inability to shift cognitive response set and would, therefore, be compatible with the theory of deficient inhibitory processes in frontostriatal diseases [32].

The correlational analysis showed that there appeared to be an association between performance on switching indices and on key neuropsychological tests known to be sensitive to frontostriatal impairment (such as the IDED set-shifting task, Stroop test and Reitan trail-making test). This consistent trend for impairment in shifting between cognitive sets demonstrated across a range of tasks further underpins the direct relevance of the dysfunctional frontostriatal circuit in the explanation of a switching deficit in verbal fluency tasks. Of interest was the trend for dissociation in the pattern of correlations of motor indices and the overall measure of cognition (MMSE), with type of switching. Motor indices correlated significantly with phonemic switching, whilst the MMSE score correlated with semantic switching. These findings may be accounted for by the common frontostriatal motor loop mediating both motor behaviour and phonemic switching in the more frontally dependant letter fluency task. Support for this view comes from Parkinson's disease patients with frontostriatal impairment who often demonstrate bradyphrenia (i.e. slowness of mental processing) in conjunction with bradykinesia (i.e. slowness of motor execution) $[25,28]$.

In summary, this longitudinal study has provided new evidence in support of the progressive impairment of switching and preservation of clustering in HD patients' performance of verbal fluency tasks. Specifically, phonemic switching alone drives verbal fluency performance and is selectively impaired, presumably due to the frontostriatal pathophysiology of HD.

\section{Acknowledgements}

This study was supported by grants from the MRC, Huntington's disease Association, Wellcome Trust and was carried out within an MRC Co-operative Group in Brain, Behaviour and Neuropsychiatry. The authors wish to thank A. Lawrence, L. Watkins, S. Rahman and R. Holt for their contribution towards patient assessment over the years.

\section{References}

[1] Audenaert K, Brans B, Van Laere K, et al. Verbal fluency as a prefrontal activation probe: a validation study using $99 \mathrm{mTc}-\mathrm{ECD}$ brain SPET. European Journal of Nuclear Medicine 2000;27:1800-8.

[2] Bachoud-Levi AC, Maison P, Bartolomeo P, et al. Re-test effects and cognitive decline in longitudinal follow-up of patients with early HD. Neurology 2001;56:1052-8.

[3] Bamford KA, Caine ED, Kido DK, et al. A prospective evaluation of cognitive decline in early Huntington's disease: functional and radiographic correlates. Neurology 1995;45:1867-73.

[4] Benton AL. Differential behavioural effects in frontal lobe disease. Neuropsychologia 1968;6:53-60.

[5] Butters N, Sax D, Montgomery K, Tarlow S. Comparison of the neuropsychological deficits associated with early and advanced Huntington's disease. Archives of Neurology 1978;35:585-9.

[6] Butters N, Wolfe J, Granholm E, Martone M. An assessment of verbal recall, recognition and fluency abilities in patients with Huntington's disease. Cortex 1986;22:11-32.

[7] Butters N, Granholm E, Salmon DP, et al. Episodic and semantic memory: a comparison of amnesic and demented patients. Journal of Clinical and Experimental Neuropsychology 1987;9:479-97. 
[8] Corcoran R, Upton D. A role for the hippocampus in card sorting? Cortex 1993;29(2):293-304.

[9] Coslett HB, Bowers D, Verfaellie M, Heilman KM. Frontal verbal amnesia. Phonological amnesia. Archives of Neurology 1991;48:94955 .

[10] Crowe SF. Dissociation of two frontal lobe syndromes by a test of verbal fluency. Journal of Clinical and Experimental Neuropsychology 1992;14:327-39.

[11] Cuenod CA, Bookheimer SY, Hertz-Pannier L, et al. Functional MRI during word generation, using conventional equipment: a potential tool for language localisation in the clinical environment. Neurology 1995;45:1821-7.

[12] Downes JJ, Roberts AC, Sahakian BJ, et al. Impaired extra-dimensional shift performance in medicated and unmedicated Parkinson's disease: evidence for a specific attentional dysfunction. Neuropsychologia 1989;27:1329-43.

[13] Folstein MF, Folstein SE, McHugh PR. Mini-mental state. A practical method for grading the cognitive state of patients for the clinician. Journal of Psychiatry Research 1975;12:189-98.

[14] Gruenewald PJ, Lockhead GR. The free recall of category examples. Journal of Experimental Psychology: Human Learning and Memory 1980;6:225-40.

[15] Huntington's Disease Collaborative Research Group. A novel gene containing a trinucleotide repeat that is expanded and unstable on Huntington's disease chromosomes. Cell 1993;72:971-83.

[16] Hedreen JC, Folstein SE. Early loss of neostriatal striosome neurons in Huntington's disease. Journal of Neuropathology and Experimental Neurology 1995;54:105-20.

[17] Hodges JR, Salmon DP, Butters N. Differential impairment of semantic and episodic memory in Alzheimer's and Huntington's diseases: a controlled prospective study. Journal of Neurology Neurosurgery and Psychiatry 1990;53:1089-95.

[18] Hodges JR, Patterson K, Oxbury S, Funnell E. Semantic dementia. Progressive fluent aphasia with temporal lobe atrophy. Brain 1992;115:1783-806.

[19] Hodges JR, Patterson K. Is semantic memory consistently impaired early in the course of Alzheimer's disease? Neuroanatomical and diagnostic implications. Neuropsychologia 1995;33:441-59.

[20] Hodges JR, Patterson K. Non-fluent progressive aphasia and semantic dementia: a comparative neuropsychological study. Journal of International Neuropsychological Society 1996;2:511-24.

[21] Huntington Study Group. Unified Huntington's disease rating scale: reliability and consistency. Movement Disorders 1996;11:136-42.

[22] Leggio MG, Silveri MC, Petrosini L, Molinari M. Phonological grouping is specifically affected in cerebellar patients: a verbal fluency study. Journal of Neurology Neurosurgery and Psychiatry 2000;69:102-6.

[23] Marder $\mathrm{K}$, Zhao $\mathrm{H}$, Myers $\mathrm{RH}$, et al. Rate of functional decline in Huntington's disease. Huntington study group. Neurology 2000;54:452-8.
[24] Martin RC, Loring DW, Meador KJ, Lee GP. The effects of lateralized temporal lobe dysfunction on formal and semantic word fluency. Neuropsychologia 1990;28:823-9.

[25] Mayeux R, Stern Y, Sano M, et al. Clinical and biochemical correlates of bradyphrenia in Parkinson's disease. Neurology 1987;37:1130-4.

[26] Mummery CJ, Patterson K, Hodges JR, Wise RJ. Generating 'tiger' as an animal name or a word beginning with $\mathrm{T}$ : differences in brain activation. Proceedings of the Royal Society of London - B Biological Sciences 1996;263:989-95.

[27] Newcombe F. Missile wounds of the brain. London: Oxford University Press, 1969.

[28] Pate DS, Margolin DI. Cognitive slowing in Parkinson's and Alzheimer's patients: distinguishing bradyphrenia from dementia. Neurology 1994;44:669-74.

[29] Perret E. The left frontal lobe of man and the suppression of habitual responses in verbal categorical behaviour. Neuropsychologia 1974;12:323-30.

[30] Reitan RM. Validity of the trail making test as an indicator of organic brain damage. Perceptual and Motor Skills 1958;8:271-6.

[31] Rich JB, Troyer AK, Bylsma FW, Brandt J. Longitudinal analysis of phonemic clustering and switching during word-list generation in Huntington's disease. Neuropsychology 1999;13:525-31.

[32] Robbins TW, Brown VJ. The role of the striatum in the mental chronometry of action: a theoretical review. Reviews in the Neurosciences 1990;2:181-213.

[33] Rosser A, Hodges JR. Initial letter and semantic category fluency in Alzheimer's disease Huntington's disease and progressive supranuclear palsy. Journal of Neurology Neurosurgery and Psychiatry 1994;57:1389-94.

[34] Stroop JR. Studies of interference in serial verbal reactions. Journal of Experimental Psychology 1935;18:643-62.

[35] Szatkowska I, Grabowska A, Szymanska O. Phonological and semantic fluencies are mediated by different regions of the prefrontal cortex. Acta Neurobiologiae Experementalis (Warsz) 2000;60:503-8.

[36] Troster AI, Fields JA, Testa JA, et al. Cortical and subcortical influences on clustering and switching in the performance of verbal fluency tasks. Neuropsychologia 1998;36:295-304

[37] Troyer AK, Moscovitch M, Winocur G. Clustering and switching as two components of verbal fluency: evidence from younger and older healthy adults. Neuropsychology 1997;11:138-46.

[38] Troyer AK, Moscovitch M, Winocur G, et al. Clustering and switching on verbal fluency: the effects of focal frontal- and temporal-lobe lesions. Neuropsychologia 1998;36:499-504.

[39] Troyer AK, Moscovitch M, Winocur G, et al. Clustering and switching on verbal fluency tests in Alzheimer's and Parkinson's disease. Journal of International Neuropsychological Society 1998;4:137-43.

[40] Wixted JT, Rohrer D. Analysing the dynamics of free recall: an integrative review of the empirical literature. Psychonomic Bulletin and Review 1994;1:89-106. 